\title{
In vitro investigation on the antiglycative and carbonyl trapping activities of hydroxytyrosol
}

\author{
Marta Navarro - Francisco J. Morales \\ 'Institute of Food Science, Technology and Nutrition, ICTAN-CSIC, 28040 Madrid, Spain
}

\begin{abstract}
Advanced glycation end products (AGEs) are involved in the aging and the development of common chronic diseases. Hydroxytyrosol (HT) and its acetate derivative (HTA) exert a significant inhibitory activity on the formation of fluorescent AGEs in bovine serum albumin glycation model systems induced by methylglyoxal (IC50 value of 0.48 and $0.58 \mu \mathrm{mol} / \mathrm{mL}$, respectively) and glucose (IC50 2.30 and 2.92 $\mu \mathrm{mol} / \mathrm{mL}$, respectively). Furthermore, HT and HTA showed a relevant carbonyl scavenging capacity toward methylglyoxal and glyoxal, which are the most potent promoters of the glycation in vivo, at molar reaction rates from 0.2 to 10 (carbonyl:phenol). However, carbonyl trapping capacity was significantly more effective against methylglyoxal (IC50 $0.19 \mu \mathrm{mol} / \mathrm{mL}$ ) than glyoxal (IC50 $0.26 \mu \mathrm{mol} / \mathrm{mL}$ ). At equimolar concentrations, the ester linkage did not significantly affect the antiglycative activity and carbonyl trapping capacity of the orthodiphenolic ring structure. Results were confirmed with the specific inhibition of the formation of the principal AGEs. Formation of carboxymethyl-lysine, argpyrimidine and carboxyethyl-lysine was significantly reduced by $61.9,71.4$ and $20.9 \%$, respectively. $\mathrm{HT}$ and its esters could be considered for upscaling studies as promising natural strategy against adverse consequences of protein glycation.
\end{abstract}

KEYWORDS: Hydroxytyrosol - Antiglycative activity · Advanced glycation end products Carboxymethyl-lysine $\cdot$ Carboxyethyl-lysine $\cdot$ Argpyrimidine.

ABBREVIATIONS: AGEs Advanced glycation end products; GLC $d(+)$-Glucose; BSA Bovine serum albumin; MGO Methylglyoxal; GO Glyoxal; AG Aminoguanidine; 5-MQ 5-Methylquinoxaline; NBT Nitrotetrazolium blue chloride; OPD o-Phenyldiamine; OPA o-Phthaldialdehyde; HFBA Heptafluorobutyric acid; HT Hydroxytyrosol; HTA Hydroxytyrosol acetate; PM Pyridoxamine; CML $\varepsilon$ $\mathrm{N}$-carboxymethyl-I-lysine; $\quad \mathrm{CEL} \quad \varepsilon-\mathrm{N}$-carboxyethyl-I-lysine; $\quad \operatorname{ArgP} \quad$ ArgPyrimidine; 2-MQ 2Methylquinoxaline; Q Methylquinoxaline; PBS Saline phosphate buffer; HPLC High-performance liquid chromatography

\section{INTRODUCTION}

Glycation occurs physiologically in the course of aging with the formation of advanced glycation end products (AGEs) when a free amino group of protein interacts with the carbonyl group of glucose [I]. In addition, dicarbonyl compounds, such as methylglyoxal (MGO) and glyoxal (GO), are also formed from the sugar degradation being key intermediates in AGEs formation [2]. Human plasma levels for GO and MGO have been estimated to be in the $0.3-1.5 \mu \mathrm{mol} / \mathrm{L}$ range, and their concentrations are increased in diabetes and chronic renal failure [3,4]. AGEs are a group of complex and heterogeneous products with different fluorescent and non-fluorescent structures, such as $N \varepsilon$-carboxymethyl-lysine (CML), NEcarboxyethyl-lysine (CEL), pentosidine, argpyrimidine (ArgP), glyoxal lysine dimers, or methylglyoxal lysine dimmers among others $[1,5,6]$. AGEs that accumulate in tissue altering irreversibly the functionality and protein structure by binding to certain amino acids such as lysine and arginine are structurally modified [2]. Therefore, AGEs might damage the tissues and organs underlying inflammation through specific receptor, and its accumulation is accelerated in diabetes, neurodegenerative and autoimmune diseases. AGEs have been associated with the process of normal aging and the 
development of common chronic diseases such as Alzheimer's disease, atherosclerosis or diabetes and its numerous long-term complications [1, 7].

The high incidence of these chronic diseases and the importance to maintain a healthy aging have caused that research focuses on the search for AGE inhibitors [8]. Initially, aminoguanidine (AG) was studied as synthetic AGE inhibitors, but due to toxic side effects it was discharged. Later, compounds from a natural source such as pyridoxamine, quercetin, curcumin, creatine or carnitine were considered as the most appropriate alternative [6, 9, 10]. Nowadays, certain bioactive phytochemicals are particularly relevant as natural AGE inhibitors since phenolic compounds have redox activity which could contribute to the inhibition of glycation process [II]. This hypothesis is supported by clinical studies where the development of type II diabetes might be ameliorated by natural antioxidants [12]. However, the specific biological consequences of the dietary AGEs are still under debate [13].

Hydroxytyrosol (HT, 2-(3,4-dihydroxyphenyl) ethanol) is an orthodiphenolic compound present in our diet, and in pharmacological preparations, as a secoiridoide derivative in free form or as an acetate ester. HT is essential component of oleuropein, present in olive oil and mill waste in appreciable quantities [14]. Dietary intake of olive oil polyphenols has been estimated to be around $9 \mathrm{mg}$ (within 2550 olive oil/day), where at least I $\mathrm{mg}$ of them is derived from free HT and $8 \mathrm{mg}$ related to their elenolic esters and oleuropein [15]. Chemical structure of HT and its derivative, hydroxytyrosol acetate (HTA) (3,4-dihydroxyphenylethyl acetate), are shown in Fig. I. Studies of HT in vivo and in vitro have shown various biological activities with potential beneficial health effects. For instance, it has been associated with anti-inflammatory, hypocholesterolemic, neuroprotective, antibacterial, cardioprotective, anticancer and insulin-stimulatory activities [14, 16]. Previous studies have linked former effects with the antioxidant activity of orthodiphenolic compounds. In vitro and in vivo studies proved that HT is a potent scavenger of free radicals and could exert an indirect protection by modulating the endogenous antioxidant defense system [15]. Although most of the studies refer to HT, it was found that HTA presents rather similar antioxidant activity than HT [17]. Restraint of oxidative stress by HT and prevention of glucose autooxidation also allowed performing a protection against certain metabolic diseases such as diabetes and its complications [17-19]. However, no alternative mechanisms for the antiglycative activity of HT have been elucidated apart of its antioxidant and antiradical properties. Recently, a number of in vitro studies have pointed out the direct relationship between the major constituents in olive leaf [20], or olive oil wastewater [2I] and the inhibition of fluorescent AGEs and the mitigation of the carbonyl stress by trapping reactive dicarbonyls [22]. However, those studies just focused on the inhibition of the formation of fluorescent derived AGEs, but there is no information of the specific inhibition on the formation of AGEs structures as formed in vivo.

This study aimed to investigate the antiglycative activity of HT and its acetate derivative directly on the formation of fluorescent AGEs by applying different in vitro models, but also through the inhibition of major AGEs, such as CML, CEL and ArgP. Additionally, ability of HT and HTA against a direct trapping of MGO and GO, and the inhibition of the formation of fructosamine adducts were also evaluated.

\section{MATERIALS AND METHODS}

\section{Chemical and materials}

$d(+)$-Glucose (GLC), bovine serum albumin (BSA), MGO (40\% aqueous solution), GO (40\% aqueous solution), aminoguanidine (AG), 5-methylquinoxaline (5-MQ), nitrotetrazolium blue chloride (NBT), ophenyldiamine (OPD), Na-acetyl-I-lysine, o-phthaldialdehyde (OPA), sodium borohydride, perfluoropentanoic acid (purity $>97 \%$ ), heptafluorobutyric acid (HFBA) and standards of phenolic acids were provided by Sigma (St. Louis, MO, USA). HT and HTA (purity $>99 \%$ ) were acquired from Seprox Biotech (Madrid, Spain). Pyridoxamine (PM) was acquired from Fluka Chemical (Madrid, Spain). Sodium dihydrogen phosphate monohydrate, sodium carbonate anhydrous, glacial acetic acid and high- 
performance liquid chromatography (HPLC)-grade methanol were purchased from Merck (Darmstadt,

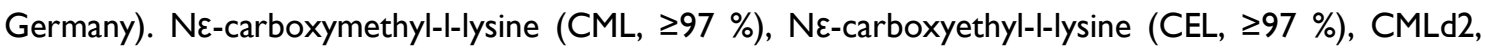
CEL-d4 and argpyrimidine (ArgP) were obtained from PolyPeptide Laboratories (Strasbourg, France). The Milli-Q water was obtained by an Elix3 water purification system coupled to a Milli-Q Advance 10 module (Millipore, Molsheim, France). All other chemicals were of analytical grade and supplied by Panreac Química (Barcelona, Spain).

\section{In vitro glycation assay with bovine serum albumin induced by methylglyoxal and glucose}

Two glycation models on BSA, one induced by MGO (BSA-MGO assay), and the other induced by glucose (BSA-GLC assay), were carried out as described by Mesias et al. [23]. The BSA solution (0.525 $\mu \mathrm{mol} / \mathrm{mL}$ ) was prepared in phosphate buffer $(0.01 \mathrm{~mol} / \mathrm{L}, \mathrm{pH} 7.4)$, containing $0.1 \mathrm{~g} / \mathrm{mL}$ sodium azide and penicillin $\mathrm{G}(0.05 \mathrm{~g} / \mathrm{mL})$ to ensure aseptic conditions and EDTA $(0.026 \mathrm{~g} / \mathrm{mL})$ to prevent metal-catalyzed oxidation. The MGO stock solution $(5.55 \mathrm{I} \mu \mathrm{mol} / \mathrm{mL})$ and glucose stock solution $(975.381 \mu \mathrm{mol} / \mathrm{mL})$ were prepared in phosphate buffer ( $0.1 \mathrm{~mol} / \mathrm{L}$, $\mathrm{pH}$ 7.4). In the BSA-MGO assay, $200 \mu \mathrm{L}$ of BSA solution was incubated with $400 \mu \mathrm{L}$ of $\mathrm{MGO}$ solution $(5.55 \mathrm{I} \mu \mathrm{mol} / \mathrm{mL})$ at $37^{\circ} \mathrm{C}$ for 14 days in the presence of $100 \mu \mathrm{L}$ of the target compound. In the BSA-GLC assay, $200 \mu \mathrm{L}$ of BSA solution was incubated with 400 $\mathrm{MI}$ of GLC solution at $37{ }^{\circ} \mathrm{C}$ for $2 \mathrm{I}$ days in the presence of $100 \mu \mathrm{L}$ of the target compound. HT, HTA and phenolic acids were dissolved in methanol/water $(60 / 40, \mathrm{v} / \mathrm{v})$, and concentrations from 0.058 to 5.8 $\mu \mathrm{mol} / \mathrm{mL}$ were prepared. In both assays, a blank without target compounds and a positive control with AG $(7.694 \mu \mathrm{mol} / \mathrm{mL})$ were incubated at the same conditions. A portion of each system at the initial time was kept at $-80{ }^{\circ} \mathrm{C}$ until measurement. In parallel, standards (from 0.058 to $5.8 \mu \mathrm{mol} / \mathrm{mL}$ ) were dissolved in phosphate buffer ( $0.1 \mathrm{~mol} / \mathrm{L}, \mathrm{pH} 7.4$ ) and incubated at $37^{\circ} \mathrm{C}$ for 14 days (BSA-MGO assay) or $2 \mathrm{I}$ days (BSA-GLC) in order to measure their intrinsic fluorescence.

The fluorescence intensity is as an index of the formation of fluorescent AGEs. A Synergy ${ }^{\mathrm{TM}} \mathrm{MX}$ multimode microplate spectrophotometer (BioTek Instruments, Winooski, VT, USA) was used. The formation of AGEs was characterized by fluorescence with excitation and emission at 340 and $420 \mathrm{~nm}$ for the BSA-MGO assay and 360 and $420 \mathrm{~nm}$ for the BSA-GLC. The intrinsic fluorescence of the standards incubated under the same conditions was subtracted to the global fluorescence in each BSAMGO or BSA-GLC system. The percentage inhibition of AGEs formation was calculated according to the following equation: The concentration required to inhibit the glycation by $50 \%$ (IC50) was calculated from the dose-response curve using the Microsoft Excel computer software.

\section{Fructosamine adduct assay}

Procedure was carried out as described by Baker et al. [24] with slight modifications. The extent of glycation on BSA was determined by measuring the reducing activity of NBT for Amadori compound in alkaline solution. Under these conditions, fructosamine has the ability to reduce NBT to tetrazinolyl radical (NBT+) forming a colored compound called monoformazan. The samples from the BSA-GLC assay were used for the determination. Thereby, $50 \mu \mathrm{L}$ of sample was mixed with $450 \mu \mathrm{L}$ of NBT and the mixture was incubated in darkness at $37^{\circ} \mathrm{C}$ for $60 \mathrm{~min}$. Then, in each well of the microplate were added $100 \mu \mathrm{L}$ of mixture and $100 \mu \mathrm{L}$ of sodium carbonate buffer $(0.1 \mathrm{~mol} / \mathrm{L}, \mathrm{pH} 10.35)$ except wells of blank that only contented $200 \mu \mathrm{L}$ of sodium carbonate buffer $(0.1 \mathrm{~mol} / \mathrm{L}, \mathrm{pH} 10.35)$. The presence of fructosamine was characterized by the absorbance of monoformazan at $530 \mathrm{~nm}$ using a BioTek microplate spectrophotometer. The inhibition of the fructosamine formation, expressed as percentage, was calculated taking into consideration the absorbance in the presence/absence of the inhibitor.

\section{Determination of reactive lysine in BSA using OPA}

The reactive lysine residues in BSA were determined as described by Goodno et al. [25]. BSA solution $(200 \mu \mathrm{L}, 35 \mathrm{mg} / \mathrm{mL}$ in phosphate buffer $0.01 \mathrm{~mol} / \mathrm{L}, \mathrm{pH} 7.4)$ was incubated at $37^{\circ} \mathrm{C}$ for I, 2 and 3 days in the absence or the presence of $\mathrm{HT}(100 \mu \mathrm{L}, 4.112 \mu \mathrm{mol} / \mathrm{mL}$ in $60: 40$ methanol/ water). The samples (20 
$\mu \mathrm{L})$ were mixed with $200 \mu \mathrm{L}$ of protein buffer (50\% buffer borate $(0.1 \mathrm{M}, \mathrm{pH} 10), 10 \%$ SDS (20\%, $\mathrm{w} / \mathrm{w}$ ) and $40 \%$ water). Na-acetyl-lysine in formic acid $0.1 \%$, at various concentrations was used for calibration (1000-50 $\mu \mathrm{M})$. The presence of reactive lysine was measured by fluorescence of at $360 / 460$ $\mathrm{nm}$ (excitation/ emission wavelength) and expressed as percentage of amino acid blockage.

\section{Evaluation of direct MGO and GO trapping capacity}

Direct MGO and GO trapping capacity was determined as described by Mesias et al. [23] with modifications. The MGO solution $(5.551 \mu \mathrm{mol} / \mathrm{mL})$ or $\mathrm{GO}$ solution $(5.686 \mu \mathrm{mol} / \mathrm{mL})$ was dissolved in phosphate buffer ( $0.1 \mathrm{~mol} / \mathrm{L}, \mathrm{pH} 7.4$ ), $5-\mathrm{MQ}$ (internal standard, I mg/mL) in $100 \mu \mathrm{L}$ of MGO or GO solution. The mixture was incubated at $37{ }^{\circ} \mathrm{C}$ up to $168 \mathrm{~h}$. After that, $200 \mu \mathrm{L}$ of OPD solution was added, rapidly vortexed for $5 \mathrm{~s}$ and was kept in darkness for $30 \mathrm{~min}$ where the derivatization reaction of unreacted dicarbonyl to corresponding quinoxaline derivative was complete. The quantification of $Q, 2-$ MQ and 5-MQ was carried out on Shimadzu HPLC system equipped with an LC-20AD pump, an SILIOADvp autosampler, a CTO-IOASVP oven and an SPD-M20A diode array detector. Chromatographic separation was performed on a Mediterranean- Sea-ODS2 column (I50 mm $\times 3 \mathrm{~mm}, 5 \mu \mathrm{m}$; Teknokroma, Barcelona, Spain). The injection volume was $10 \mu \mathrm{L}$, and the flow rate was $0.5 \mathrm{~mL} / \mathrm{min}$. The total run time was $7 \mathrm{~min}$ in isocratic conditions (mobile phase of $5 \mathrm{~mL} / \mathrm{L}$ acetic acid/methanol, 50:50 v/v). The chromatograms were recorded at $315 \mathrm{~nm}$ being the retention times of Q, 2-MQ and 5-MQ, 3.4, 4.I and $5.6 \mathrm{~min}$, respectively. The amount of unreacted MGO was calculated from the ratio of 2-MQ and 5$M Q$ as compared with control at the initial time. The amount of unreacted $G O$ was calculated from the ratio of $\mathrm{Q}$ and 5-MQ as compared with control at the initial time. The percentage of inhibition of MGO or $\mathrm{GO}$ was calculated with the next formula. The concentration required to inhibit the glycation by 50 $\%$ (IC50) was obtained from the dose-response curves using Microsoft Excel computer software

\section{Determination of CML and CEL by LC-MS/MS}

The formation of CML and CEL was determined as described by Niquet-Léridon and Tessier [26] with minor modifications. Sample ( $\mathrm{mL}$, containing $10 \mathrm{mg}$ of protein) was incubated with $1.5 \mathrm{~mL}$ of sodium borate $(0.2 \mathrm{M}, \mathrm{pH} 9.2)$ and I $\mathrm{mL}$ of sodium borohydride (I $\mathrm{M}$ in $0.1 \mathrm{M} \mathrm{NaOH})$ for $4 \mathrm{~h}$ at room temperature. Then, samples were hydrolyzed with $5 \mathrm{~mL}$ of $\mathrm{HCl}$ (final concentration of $6 \mathrm{M}$ ) for $20 \mathrm{~h}$ at $110{ }^{\circ} \mathrm{C}$, filtered and $500 \mu \mathrm{L}$ was dried in a Speed-Vac concentrator (ThermoFisher Scientific, Courtaboeuf, France). Finally, residue was reconstituted in $1 \mathrm{~mL}$ of NFPA $5 \mathrm{mM}$ (containing $0.1 \mathrm{~mL}$ of CML-d2 or CEL-d4 at I $\mu g / \mathrm{mL}$ ). LC-MS/MS was performed with an Agilent 1200 HPLC system (Agilent Technologies, Palo Alto, CA, USA) coupled to a triple quadrupole Agilent (G64IOB) via electrospray ionization operating in positive mode. Sample (10 $\mu \mathrm{L})$ was injected on Hypercarb column $(100 \mathrm{~mm} \times 2.1$ $\mu \mathrm{m}, 5 \mu \mathrm{m}$, ThermoFisher Scientific). The flow rate was $0.5 \mathrm{~mL} / \mathrm{min}$, and the isocratic elution was applied using a mobile phase of $5 \mathrm{mM}$ NFPA / acetonitrile $(95: 5 \mathrm{v} / \mathrm{v})$. The product ion at $\mathrm{m} / \mathrm{z} 84$ was used for quantification of CML (m/z 205), CML-d2 (m/z 207), CEL (m/z 219) and CEL-d4 (m/z 223). The ratio of response factor of CML or CEL to that their respective labeled internal standards was used for calculation of CML or CEL in samples. Data were processed using Masshunter Data Acquisition and MassHunter Qualitative Analysis (Agilent Technologies). Results were expressed as $\mu \mathrm{mol}$ CML or CEL Ig protein.

\section{Determination of argpyrimidine by LC-fluorescence}

Sample preparation was the same as described above for lysine-derived AGEs until obtaining the freezedried protein hydrolysate as described by Wilker et al. [27]. The analysis of ArgP (10 $\mu \mathrm{L}$ sample injection) was carried out on a Prominence HPLC system with an RF-20A XS fluorescence detector (Shimadzu Corporation, Kyoto, Japan). Sample was eluted onto a Mediterranean-Sea-ODS2 column (250 $\mathrm{mm} \times 4 \mathrm{~mm}, 5 \mu \mathrm{m}$; Teknokroma, Barcelona, Spain) at a flow rate of $0.8 \mathrm{~mL} / \mathrm{min}$ under gradient elution. The solvent system gradient was of HFBA ( $(\mathrm{mL} / \mathrm{L})$ as solvent $A$ and ACN $(500 \mathrm{~mL} / \mathrm{L})$ containing HFBA $(\mathrm{I} \mathrm{mL} / \mathrm{L})$ as solvent $\mathrm{B}$. The following multistep linear gradient was applied: $0 \mathrm{~min}, 20 \% \mathrm{~B} ; 25 \mathrm{~min}, 100 \%$ 
B; $26 \mathrm{~min}, 100 \% \mathrm{~B}$; and 27-37 min, $20 \% \mathrm{~B}$. ArgP was detected at 335 and $385 \mathrm{~nm}$ for excitation and emission wavelength, respectively, and eluted at $15.1 \mathrm{~min}$. Calibration was carried out in the range $0.001-0.01 \mathrm{mg} / \mathrm{mL}$ with pure standard. Results were expressed as $\mu \mathrm{mol} \mathrm{ArgP} / \mathrm{g}$ protein.

\section{Statistical analysis}

Data were analyzed using the Statgraphics Centurion XV Statistical program (Herndon, VA, USA). Homogeneity of variances was evaluated by the test of Levene. Analysis of variance was performed using ANOVA procedures followed by a Bonferroni test or Tamhane test when the variances were homogeneous or non-homogeneous, respectively. Differences were considered significant at $p<0.05$.

Data from three independent assays were expressed as the mean value \pm SD.

\section{RESULTS AND DISCUSSION}

The antiglycative activity of HT and HTA was evaluated by measuring the inhibition of the formation of fluorescent AGEs in a model of glycation with BSA under physiological conditions. Accumulation of AGE-specific fluorescence is a general measure of overall protein glycation damage, and it is a widely used tool for glycation research in in vitro and in vivo studies [6]. Since HT and HTA were dissolved in a methanolic solution $(60 \% \mathrm{v} / \mathrm{v})$, the lack of potential interferences in the glycation models was evaluated. Different proportions of methanol:water up to $100 \%$ after 14 or 21 days of incubation at $37^{\circ} \mathrm{C}$ for BSA-MGO and BSA-GLC systems, respectively, were tested. In addition, the intrinsic fluorescence of HT and HTA was residual, accounting for $<5 \%$ of the total fluorescence in the glycation models.

The antiglycative capacity of $\mathrm{HT}$ and its acetate derivative in a range from 0.405 to $8.282 \mu \mathrm{mol} / \mathrm{mL}$ were assayed in the BSA-GLC $\left(2 \mathrm{I}\right.$ days $\left./ 37^{\circ} \mathrm{C}\right)$ and BSA-MGO $\left(14\right.$ days $\left./ 37^{\circ} \mathrm{C}\right)$ systems. Glycation reaction in the BSA-GLC system proceeds slower than the BSA-MGO system since autoxidation of glucose to form dicarbonyl compounds is minimized in the presence of EDTA. Another limiting step in the BSAGLC system is the conversion of glucose to its open-chain form to condensate with the amino residues [2]. Figure 2 shows the percentage of inhibition of the formation of fluorescent AGEs related to the molar ratio between the glycative stressor and the orthodiphenolic compound. Both HT and HTA exerted a dosedependent antiglycative activity in BSA-MGO and BSA-GLC systems. Maximum inhibition (98.6\%) was reached at $4.112 \mu \mathrm{mol} / \mathrm{mL}$ in the BSA-MGO system (Fig. 2a), corresponding to a molar ratio (MGO:phenolic compound) of 0.8. At lower molar ratio, the activity was kept without significant differences $(p<0.05)$ among samples. The potency of the antiglycative capacity of HTA was similar to that $\mathrm{HT}$ at equimolar concentration of MGO, although response of HTA was significantly lower than HT at $0.405 \mu \mathrm{mol} / \mathrm{mL}$ (8 molar ratio MGO:phenolic compound). AG was used as positive control since it inhibits AGEs formation by reacting with carbonyl groups of reducing sugars, Amadori products and dicarbonyl intermediates [6]. AG reached $99.6 \%$ of inhibition at the same molar ratio MGO:AG. IC50 values of 0.48 and $0.58 \mu \mathrm{mol} / \mathrm{mL}$ were calculated for HT and HTA in the BSA-MGO system, respectively. In the BSA-GLC system (Fig. 2b), although a similar trend was observed, the antiglycative activity of HT was significantly higher than HTA at higher molar ratio with GLC. Additionally, IC50 value of 2.30 and $2.92 \mu \mathrm{mol} / \mathrm{mL}$ was calculated for HT and HTA, respectively, for their antiglycative activity in the BSA-GLC system.

The extent of the sugar-induced modifications was further evaluated in the BSA-GLC system by the formation of the Amadori products using the fructosamine assay. In this assay, glucosone is the primary carbohydrate oxidation product formed from the Amadori compounds. In this sense, fructosamine assay is an efficient tool to identify at which level of the glycation cascade is acting our target compounds. Table I shows the inhibition of the formation of the Amadori products in the presence of different concentrations of HT and HTA $(0.405-8.282 \mu \mathrm{mol} / \mathrm{mL})$. Both HT and HTA are able to prevent the formation of the Amadori product but in an efficiently manner. Results are correlated with the antiglycative effect of $H T$ and HTA in the BSA-GLC assay. $H T$ was significantly more effective than HTA 
in the prevention of the formation of Amadori products at a molar ratio (GLC:phenolic compound) of 600 , whereas there were no differences at lower molar ratio since a complete inhibition was already reached. These results on fructosamine could explain the higher activity of HT than HTA exerted in the inhibition of the fluorescent AGEs in the BSA-GLC assay.

The protection of reactive lysine residues in the protein by HT was investigated. HT was able to protect the modification of the 24.3, 39.7 and $45.1 \%$ of amino group in just I, 2 and 3 days of incubation, respectively. Recently, Navarro and Morales [22] shown that the reactivity of orthodiphenolic compounds against MGO and GO was effective enough to compete with free lysine, arginine and histidine and subsequently to prevent the formation of carbonylderived AGEs. However, the mechanism of reaction was not concluded. Our results pointed out that ortodiphenolic compounds not only could mitigate the extension of the glycation process by reducing the formation of the Amadori products but also by protecting the lysine residues to initiate the glycation reaction.

In the BSA-MGO system, our results showed the protective effect of $\mathrm{HT}$ on the formation of the fluorescent AGEs at similar molar ratio with MGO. The evaluation of the specific scavenging capacity of inhibitors toward dicarbonyl compounds gives a complementary view of the potential antiglycative activity of target compounds, and it can be used as an effective strategy to prevent protein modifications and AGEs formation [8, 28, 29]. The role of dicarbonyl compounds was further investigated in MGO and GO trapping model systems (Fig. 3) at different molar ratios (dicarbonyl:phenolic compound). Both HT and HTA displayed similar dicarbonyl trapping activity against GO regardless the molar ratio (Fig. 3b). However, significant differences between HT and HTA were found at molar ratio higher than I for trapping MGO (Fig. 3a). Dicarbonyls and particularly MGO and GO are key promoter in the glycation process [30], and they are important precursors in the formation of AGEs [I, II]. Dicarbonyls are formed from oxidative reaction to sugars, Schiff bases, Amadori products and lipid oxidation. Then, dicarbonyls irreversibly and progressively modify proteins overtime and yield AGEs which are contributing to the complications associated with aging. These modifications involve lysine residues, but arginine is predominantly modified with the formation of fluorescent compounds [5]. Our results on direct dicarbonyl trapping are in line with the results obtained for the inhibition of fluorescent AGEs in the BSA-MGO assay shown in Fig. 2a.

Additionally, the trapping capacity, expressed as IC50, of different structure-related phenolic compounds (gallic acid, caffeic acid, protocatechuic acid, chlorogenic acid, sinapinic acid, ferulic acid, gentisic acid and P-coumaric acid) is given in Table 2 . Results confirm the promising dicarbonyl trapping ability of $\mathrm{HT}$, and HTA as compared with other phenolic acids. It has been described that the hydroxyl groups in the phenyl ring have electron-donating properties and enhance the reactivity of MGO by just nucleophilic addition. Navarro and Morales [22] identified two major adducts during the incubation of HT with MGO at physiological conditions. In order to gain more insight into the dicarbonyl trapping abilities of HT and HTA, the kinetic of the trapping over time was investigated. Figure 4 shows the time course of the disappearance of dicarbonyl compounds (expressed as MGO or GO trapping capacity) in the reaction mixtures ( $100 \mathrm{mM}$ PBS, $37^{\circ} \mathrm{C}$ ) at the same dicarbonyl:phenolic compound molar ratio. PM was used as a positive control of the reaction, and HT and HTA behave as PM did (data not shown). The kinetic of $\mathrm{HT}$ and HTA was similar, time dependent and progressively is reaching a maximum activity at $168 \mathrm{~h}$ of incubation (94.6 and $95.1 \%$, respectively). Again, it was observed that HT and HTA were quantitatively less effective for trapping GO as compared with MGO at any time of incubation.

The antiglycative properties of HT were further investigated on the specific inhibition of the formation of the most representative AGEs (Fig. I). CML is the most abundant non-fluorescent AGEs and together with CEL has been used as markers of the extension of the glycation in foods and physiological systems $[1,2,30]$. In our BSA-GLC glycation system that contains EDTA, CML is basically formed by the 
condensation of glucose with the $\varepsilon$-amino group of lysine, where the Amadori rearrangement product fructosyllysine is unstable intermediate and undergoes oxidation to form CML. Then a BSA-GO model was carried out to enhance specifically the formation of CML. In contrast, the system BSA-MGO was used for the determination of CEL and ArgP. ArgP is the major fluorescent AGEs result of the reaction between two molecules of MGO and arginine [5], and CEL is a lysine-MGO-derived AGE [3]. In this investigation was not included pentosidine since it was not defined a model system with ribose. In the investigation with CML, CEL and ArgP, the incubation time of the glycation models was settled at 7 days for BSA-MGO and BSA-GO systems since the maximum concentration of these AGEs was already reached at that time. BSA-GO system was prepared in similar way to BSA-MGO.

The CML formation in the BSA-GO system (7 days of incubation at $37^{\circ} \mathrm{C}$ ) is shown in Fig. $5 \mathrm{a}$. AG was used as a positive control at the same molar ratio than $\mathrm{HT}$. The formation of CML was significantly reduced in the presence of $\mathrm{HT}$ at all the concentration assayed, being significantly stronger than AG at the highest molar ratio with GO. CEL is formed in low amounts in the BSA-MGO system, and AG is able to inhibit its formation very effectively in a dose-response manner. In regard to the formation of CEL (Fig. 5b), it was clearly observed a dose-dependent effective mitigation by AG, even at the highest MGO:AG molar ratio. However, HT was not efficient enough to impair the formation of CEL where only a $20.9 \%$ of inhibition is reached at equimolar concentration. As described by Navarro and Morales [22], arginine is the preference site of reaction for MGO, followed by histidine, and lysine is less prone to react with MGO that it could explain the low rate of formation of CEL. As expected, the arginineMGO derived AGE ArgP was largely formed in the BSA-MGO (Fig. 5c) and ArgP formation is largely dependent on the presence of $A G$ and $\mathrm{HT}$. For both compounds, the formation of ArgP was significantly reduced to 47.4 and $49.2 \%$ for $A G$ and $H T$, respectively, as compared with the control at the highest MGO molar ratio. It is noteworthy to mention that the formation of $\mathrm{ArgP}$ at equimolar concentration was completely impaired by AG and significantly reduced at $71.4 \%$ by HT. Two molecules of MGO are necessary for the formation of $\mathrm{ArgP}$, and the presence of free MGO is the limiting factor in the reaction.

The protein glycation process can be divided into the early stage in which the Amadori products are formed, and the advanced stage in which Amadori products undergo a number of rearrangements to form AGEs. Our results on the fructosamine assay and blockage of reactive amino residues reveal that HT and HTA have also an inhibitory effect on the formation of the Amadori product at the early stage of the glycation. But, HT and HTA are also effective inhibitors of the advanced stage since the formation of fluorescent AGEs, CML and ArgP were effectively suppressed. Results are promising to upscale the in vitro experiments to in vivo systems since $H T$ is abundant in olive oil and efficiently absorbed $[15,16]$. In this regard, Miro-Casas et al. [18] investigated the bioavailability of HT in animals and humans administrated with virgin olive oil have demonstrated that $\mathrm{HT}$ absorption, plasmatic levels and urine excretion have a direct response to the intake. The maximum concentration of HT in plasma is reached at $32 \mathrm{~min}$ with a half-life of $2.43 \mathrm{~h}$. Nearly $98 \%$ of the HT appears in plasma as conjugated HT and its metabolites suggesting extensive intestinal/hepatic metabolism of the dietary HT [15]. Serra et al. [3I] in an intervention experiment feeding rats with olive extract shown that $\mathrm{HT}$ was absorbed and rapidly distributed $(56.8 \mathrm{nmol} / \mathrm{L}$ plasma after I h) practically to all organs through the blood stream more effectively than the luteolin derivatives $(0.68 \mathrm{nmol} / \mathrm{L}$ plasma after I h). Other study in rats supports that after an intravenously injection of $\mathrm{HT}$, it was quickly absorbed and distributed, being preferential kidney uptake. The distribution of its different metabolites depends on the enzymatic conversion, being sulfoconjugated forms, the metabolites from a sequential oxidation (3,4-dihydroxyphenylacetaldehyde, 3,4dihydroxy-phenylacetic acid, 4-hydroxy-3-methoxy-phenylacetic acid) and the methylated derivative form (4-hydroxy-3-methoxy-phenylethanol) the majority in kidney, brain, heart, liver and brain, respectively [32]. 
In conclusion, HT and its acetate derivate, HTA, were evaluated with the aim of investigating their antiglycative capacity using different in vitro models. These orthodiphenolic compounds exhibited a potent inhibitory activity on the formation of CML, and ArgP formation and very likely the carbonyl trapping capacity plays a major role. Results are in line with Navarro and Morales [22] who described the mechanism of action for trapping carbonyl species by HT due to electrophilic aromatic substitution of the orthodiphenyl ring. Additionally, the reactivity of amino residues and fructosamine formation was efficiently inhibited so it could be assumed that HT and HTA are also acting at the early stages of glycation process. This study has shown by first time that $\mathrm{HT}$ and its acetate ester act at multiple steps of the glycation process and the reactive part of the molecular structure is the orthodiphenyl ring without major relevance to the ester linkage. These in vitro results are promising and pointed out that it is realistic to define further steps in vivo before to propose HT as a natural AGEs inhibitor.

\section{COMPLIANCE WITH ETHICAL STANDARDS}

\section{CONFLICT OF INTEREST}

The authors declare that they have no conflict of interest.

\section{HUMAN AND ANIMAL RIGHTS}

This investigation does not contain studies with human or other animal subjects.

\section{COMPLIANCE WITH ETHICS REQUIREMENTS}

This article does not contain any studies with human or animal subjects.

\section{ACKNOWLEDGEMENTS}

M. Navarro thanks to the JAE program from Spanish National Research Council for granting a predoctoral contract (Ref. JAEPre073-20II). This work was funded by Projects S20I3/ ABI-3028AVANSECAL from Comunidad of Madrid and European funding from FEDER program (European Regional Development Fund) and CSIC-201370E027 (Spanish National Research Council).

\section{REFERENCES}

I. Thorpe SR, Baynes JW (2003) Maillard reaction products in tissue proteins: new products and new perspectives. Amino Acids 25:275-28I

2. Tessier FJ (2010) The Maillard reaction in the human body. The main discoveries and factors that affect glycation. Pathol Biol 58:214-219

3. Monnier VM, Sell DR, Nagaraj RH, Miyata S, Grandhee S, Odetti P, Ibrahim SA (1992) Maillard reaction-mediated molecular damage to extracellular matrix and other tissue proteins in diabetes, aging, and uremia. Diabetes 4I:36-4I

4. Odani H, Shinzato T, Matsumoto Y, Usami J, Maeda K (1999) Increase in three $\alpha$, $\beta$-dicarbonyl compound levels in human uremic plasma: specific in vivo determination of intermediates in advanced Maillard reaction. Biochem Biophys Res Commun 256:89-93

5. Shipanova IN, Glomb MA, Nagaraj RH (1997) Protein modification by methylglyoxal: chemical nature and synthetic mechanism of a major fluorescent adduct. Arch Biochem Biophys 344:29-36

6. Wu CH, Huang SM, Lin JA, Yen GC (20II) Inhibition of advanced glycation endproduct formation by foodstuffs. Food Funct 2:224-234

7. Grillo MA, Colombatto S (2008) Advanced glycation end-products (AGEs): involvement in aging and in neurodegenerative diseases. Amino Acids 35:29-36 
8. Peng $\mathrm{X}$, Ma J, Chen F, Wang M (20II) Naturally occurring inhibitors against the formation of advanced glycation end-products. Food Funct 2:289-30I

9. Löbner J, Degen J, Henle T (2015) Creatine is a scavenger for methylglyoxal under physiological conditions via formation of $\mathrm{N}$-(4-Methyl-5-oxo-I-imidazolin-2-yl)sarcosine (MG-HCr). J Agric Food Chem 63:2249-2256

10. Rajasekar P, Anuradha CV (2007) I-Carnitine inhibits protein glycation in vitro and in vivo: evidence for a role in diabetic management. Acta Diabetol 44:83-90

II. Rahbar S, Figarola JL (2003) Novel inhibitors of advanced glycation endproducts. Arc Biochem Biophys 419:63-79

12. Montonen J, Knekt P, Jarvinen R, Reunanen A (2004) Dietary antioxidant intake and risk of type 2 diabetes. Diabetes Care 27:362-366

13. Henle T (2007) Dietary advanced glycation end products-a risk to human health? A call for an interdisciplinary debate. Mol Nutr Food Res 5I(9): 1075-1078

14. Martín-Peláez S, Covas MI, Fitó M, Kusar A et al (2013) Health effects of olive oil polyphenols: recent advances and possibilities for the use of health claims. Mol Nutr Food Res 57:760-77I

15. De la Torre R (2008) Bioavailability of olive oil phenolic compounds in humans. Inflammopharmacology 16:245-247

16. De Bock M, Thorstensen EB, Derraik JGB, Henderson HV et al (20/3) Human absorption and metabolism of oleuropein and hydroxytyrosol ingested as olive (Olea europaea L.) leaf extract. Mol Nutr Food Res 57:2079-2085

17. Gordon MH, Paiva-Martins F, Almeida M (200I) Antioxidant activity of hydroxytyrosol acetate compared with that of other olive oil polyphenols. J Agric Food Chem 49:2480-2485

18. Miro-Casas E, Covas MI, Farre M, Fito M et al (2003) Hydroxytyrosol disposition in humans. Clin Chem 49(6):945-952

19. Hamden K, Allouche N, Damak M, Elfeki A (2009) Hypoglycemic and antioxidant effects of phenolic extracts and purified hydroxytyrosol from olive mill waste in vitro and in rats. Chem Biol Interact I80:421-432

20. Kontogianni VG, Charisiadis P, Margianni E, Lamari FN et al (2013) Olive leaf extracts are a natural source of advanced glycation end product inhibitors. J Med Food 16:817-822

2I. Navarro M, Fiore A, Fogliano V, Morales FJ (2015) Carbonyl trapping and antiglycative activities of olive oil mill wastewater. Food Funct 6:574-583

22. Navarro M, Morales FJ (2015) Mechanism of reactive carbonyl species trapping by hydroxytyrosol under simulated physiological conditions. Food Chem 175:92-99

23. Mesias M, Navarro M, Gökmen V, Morales FJ (2013) Antiglycative effect of fruit and vegetable seed extracts: inhibition of AGE formation and carbonyl-trapping abilities. J Sci Food Agric 93:2037-2044

24. Baker JR, Zyzak DV, Thorpe SR, Baynes JW (1994) Chemistry of the fructosamine assay: $d$ glucosone is the product of oxidation of Amadori compounds. Clin Chem 40:1950-1955

25. Goodno CC, Swaisgood HE, Catignani GL (198I) A fluorimetric assay for available lysine in proteins. Anal Biochem II5:203-2II 
26. Niquet-Léridon C, Tessier FJ (20II) Quantification of $N \varepsilon$-carboxymethyl-lysine in selected chocolate-flavoured drink mixes using high-performance liquid chromatography-linear ion trap tandem mass spectrometry. Food Chem 126:655-663

27. Wilker SC, Chellan P, Arnold BM, Nagaraj RH (200I) Chromatographic quantification of argpyrimidine, a methylglyoxalderived product in tissue proteins: comparison with pentosidine. Anal Biochem 290:353-358

28. Totlani VM, Peterson DG (2006) Epicatechin carbonyl-trapping reactions in aqueous Maillard systems: identification and structural elucidation. J Agric Food Chem 54:73 I I-73 I8

29. Lo CY, Li S, Tan D, Pan MH et al (2006) Trapping reactions of reactive carbonyl species with tea polyphenols in simulated physiological conditions. Mol Nutr Food Res 50: I I I8-I I28

30. Hellwig M, Henle T (20I4) Baking, ageing, diabetes: a short history of the Maillard reaction. Angew Chem Int Ed 53:2-16

3I. Serra A, Rubió L, Borràs X, Macià A et al (2012) Distribution of olive oil phenolic compounds in rat tissues after administration of a phenolic extract from olive cake. Mol Nutr Food Res 56:486-496

32. D’Angelo S, Manna C, Migliardi V, Mazzoni M et al (200I) Pharmacokinetics and metabolism of hydroxytyrosol, a natural antioxidant from olive oil. Drug Metab Dispos 29(I I): I492-1498 


\section{FIGURES AND TABLES}

Figure I. Structure of hydroxytyrosol (a), hydroxytyrosol acetate (b), $\varepsilon-\mathrm{N}$-carboxymethyl-L-lysine (c), $\varepsilon-\mathrm{N}$-carboxyethyl-L-lysine (d), argypyrimidine (e).

Figure 2. Antiglycative activity of hydroxytyrosol (solid bar) and hydroxytyrosol acetate (empty bar) on the formation of fluorescent AGEs in the BSA-MGO assay (a), and in the BSA-GLC assay (b). Results are expressed as mean (percentage of inhibition respect to control) \pm standard deviation for $n=4$. Different letters denote significant differences $(P<0.05)$.

Figure 3. Methylglyoxal (a) and glyoxal (b) trapping capacity of hydroxytyrosol (solid bar) and hydroxytyrosol acetate (empty bar) at different molar ratio. Results are expressed as mean \pm standard deviation for $n=4$. Different letters denote significant differences $(P<0.05)$.

Figure 4. Methylglyoxal (a) and glyoxal (b) trapping capacity of hydroxytyrosol (solid circle) and hydroxytyrosol acetate (open circle) incubated $\left(37^{\circ} \mathrm{C}\right.$ for $\left.168 \mathrm{~h}\right)$ at the equimolar conditions $(0.58 \mathrm{I}$ $\mu \mathrm{mol} / \mathrm{mL})$. Different letters denote significant differences $(P<0.05)$.

Figure 5. Effect of hydroxytyrosol (empty bar) on the inhibition of the formation of carboxymethyllysine (a), carboxyethyl-lysine (b), and argypyrimidine (c) at different molar ratio of methylglyoxal and glyoxal. Aminoguanidine was used as reference (grey bar). BLK corresponds to control (solid bar). Results are expressed as mean \pm standard deviation for $n=3$. Different letters denote significant differences $(P<0.05)$ with control. 


\section{TABLES}

Table I. Determination of the inhibition of the formation of Amadori products by the fructosamine assay by hydroxytyrosol $(\mathrm{HT})$ and hydroxytyrosol acetate $(\mathrm{HTA})$ in the range from 0.405 to $8.282 \mu \mathrm{mol} / \mathrm{L}$.

\begin{tabular}{cccc}
$\begin{array}{c}\text { Molar Ratio } \\
\text { Glucose:Phenolic }\end{array}$ & $\begin{array}{c}\text { Concentration } \\
\mu \mathrm{mol} / \mathrm{mL}\end{array}$ & $\begin{array}{c}\mathrm{HT} \\
\text { Inhibition (\%) }\end{array}$ & $\begin{array}{c}\text { HTA } \\
\text { Inhibition (\%) }\end{array}$ \\
\hline 1200 & 0.405 & $2.9 \pm 2.3 \mathrm{a}$ & $7.2 \pm 2.0 \mathrm{a}$ \\
600 & 0.811 & $66.5 \pm 7.4 \mathrm{~b}$ & $7.3 \pm 2.1 \mathrm{a}$ \\
120 & 4.112 & $100 \pm 8.0 \mathrm{c}$ & $100 \pm 2.5 \mathrm{c}$ \\
60 & 8.282 & $100 \pm 6.7 \mathrm{c}$ & $100 \pm 7.7 \mathrm{c}$ \\
\hline
\end{tabular}

${ }^{\text {a }}$ Results are expressed as mean $\pm S D$ for $n=4$.

Different letters in same column denote significant differences $P<0.05)$. 
Table 2. Methylglyoxal trapping capacity of hydroxytyrosol, hydroxytyrosol acetate, gallic acid, caffeic acid, protocatechuic acid, chlorogenic acid (CGA), sinapinic acid, ferulic acid, gentisic acid, and $p$-coumaric acid. Pyridoxamine is used as reference.

\begin{tabular}{cc} 
Standard & $\mathrm{IC}_{50}(\mu \mathrm{mol} / \mathrm{mL})$ \\
\hline Hydroxytyrosol & 0.195 \\
Hydroxytyrosol acetate & 0.204 \\
Pyridoxamine & 0.059 \\
\hline Gallic acid & 0.176 \\
Caffeic acid & 0.222 \\
Protocatechuic acid & 0.389 \\
Chlorogenic acid & 0.367 \\
Sinapinic acid & 0.892 \\
Ferulic acid & 1.648 \\
Gentisic acid & 3.179 \\
P-Coumaric acid & 2.985 \\
\hline
\end{tabular}




\section{FIGURE 1}

a)<smiles>OCCc1ccc(O)c(O)c1</smiles>

c)<smiles>NC(CCCCNCC(=O)O)C(=O)O</smiles>

b)<smiles>CC(=O)OCCc1ccc(O)c(O)c1</smiles>

e)<smiles>CC(NCCCCC(N)C(=O)O)C(=O)O</smiles><smiles>Cc1nc(NCCCC(N)C(=O)O)nc(C)c1O</smiles> 
FIGURE 2

a

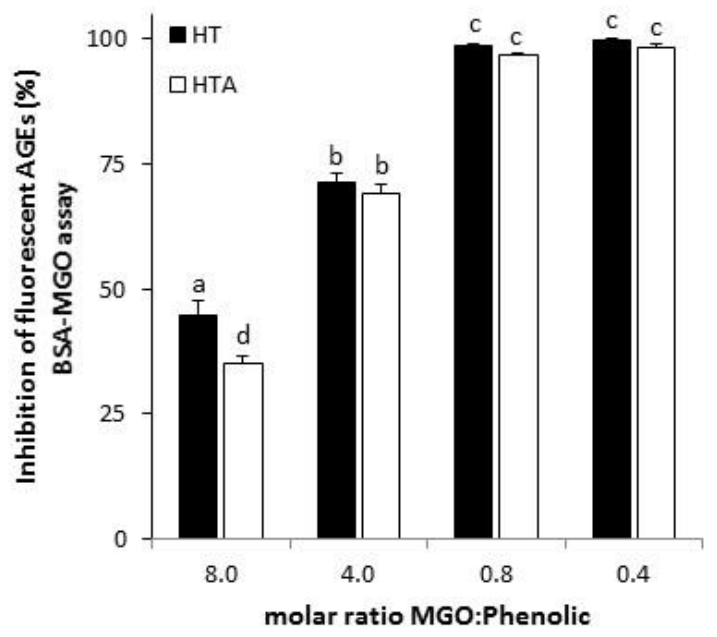

b

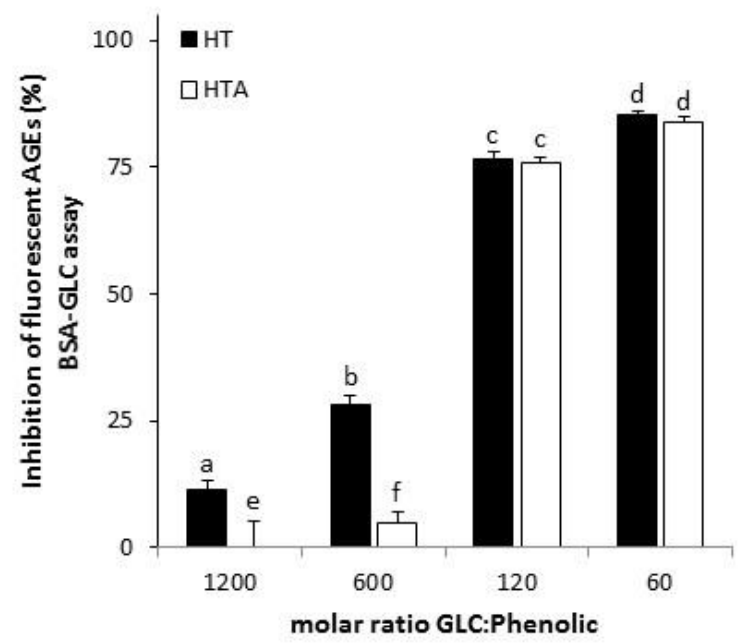


FIGURE 3

a

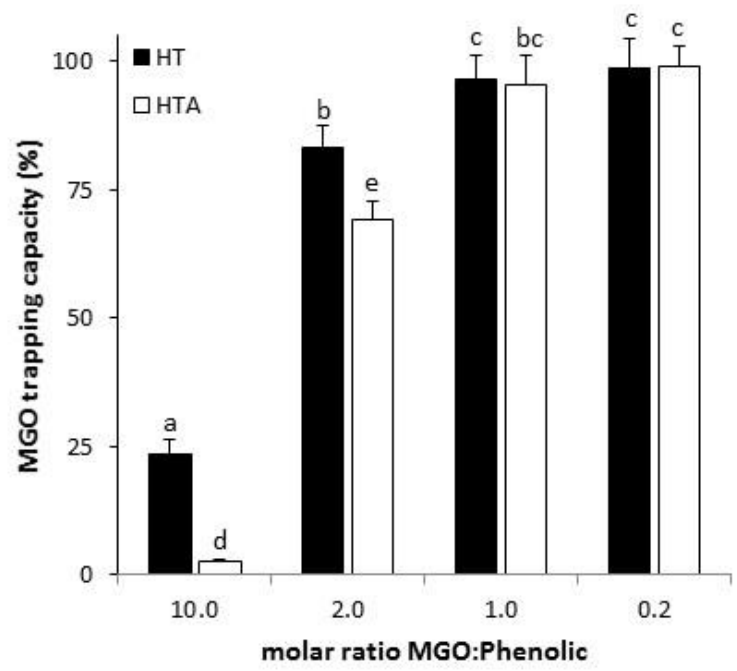

b

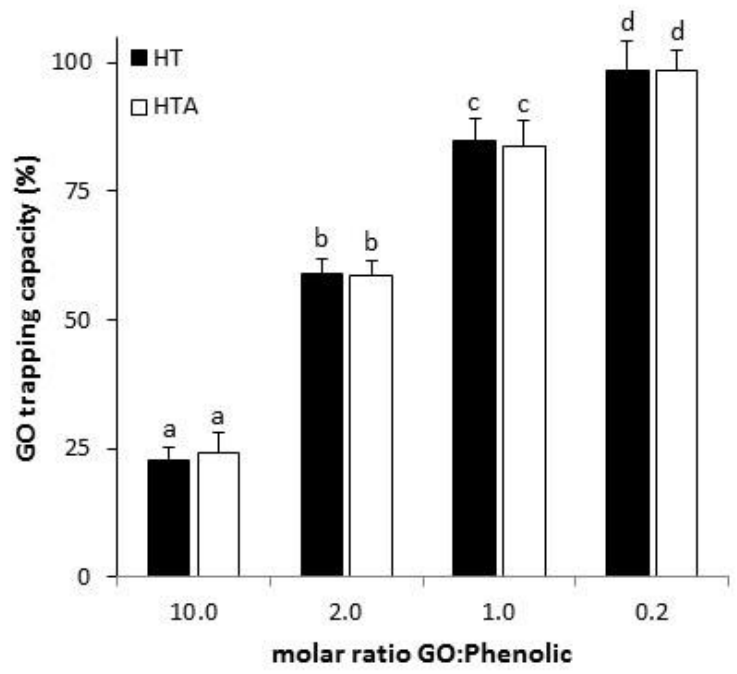




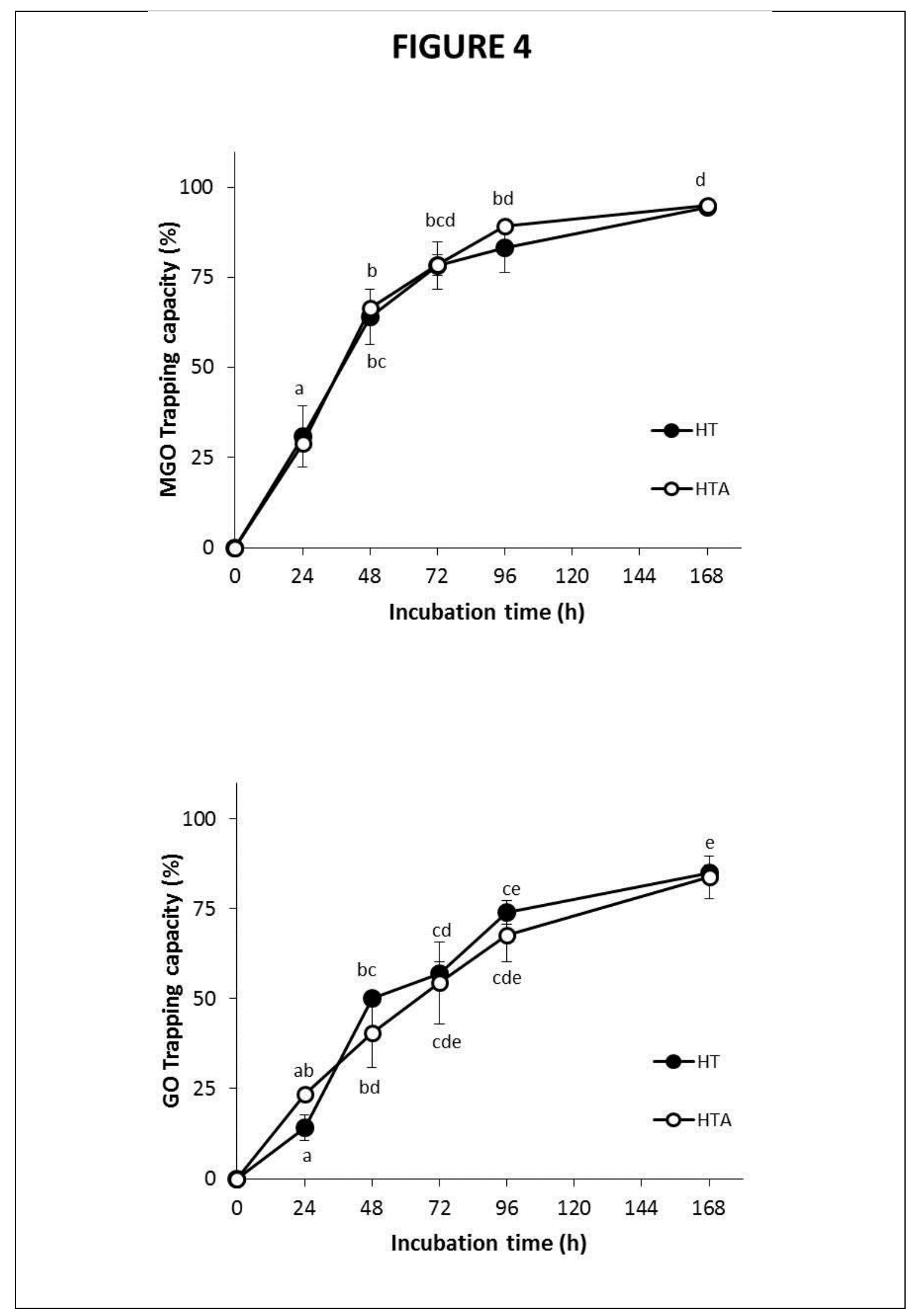




\section{FIGURE 5}

a

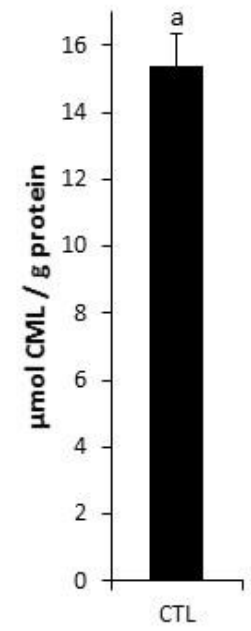

b
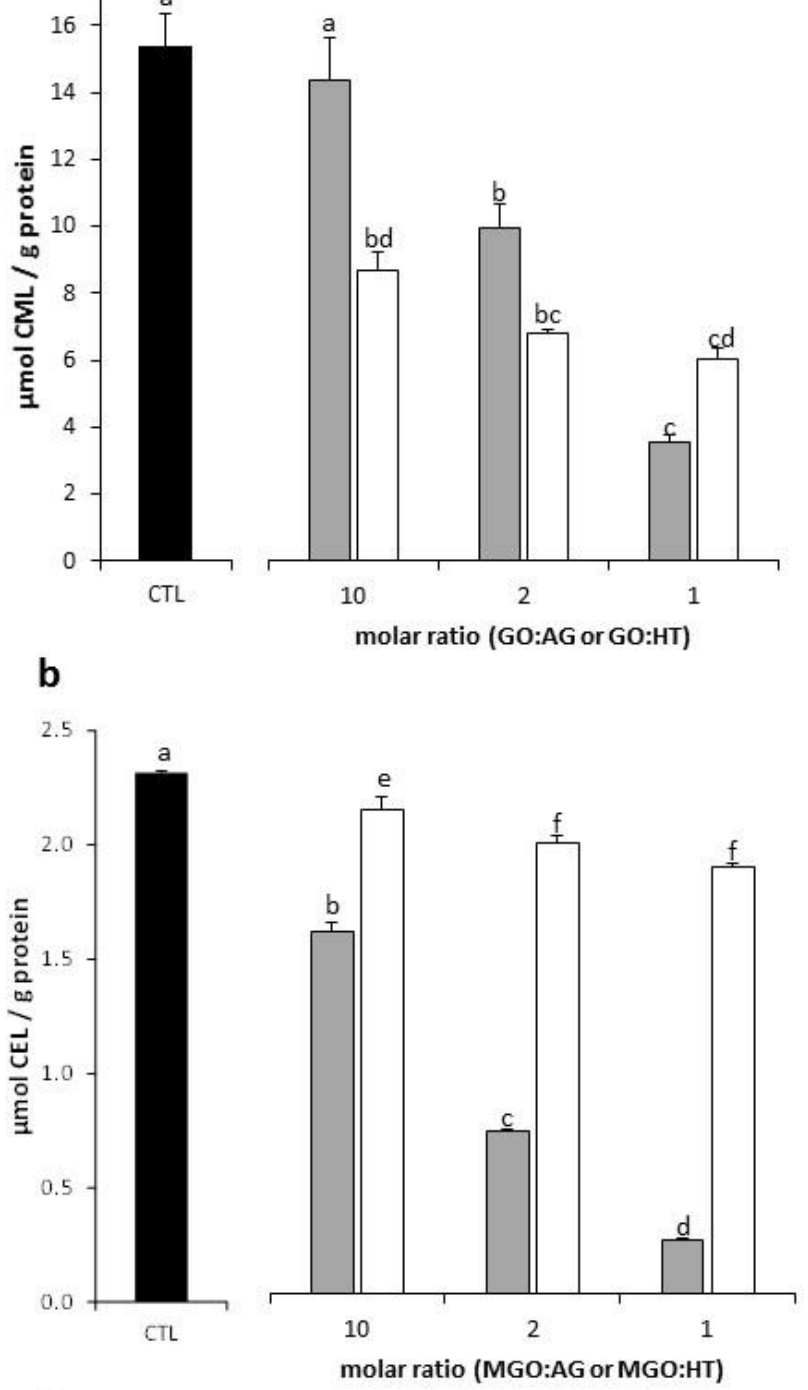

c

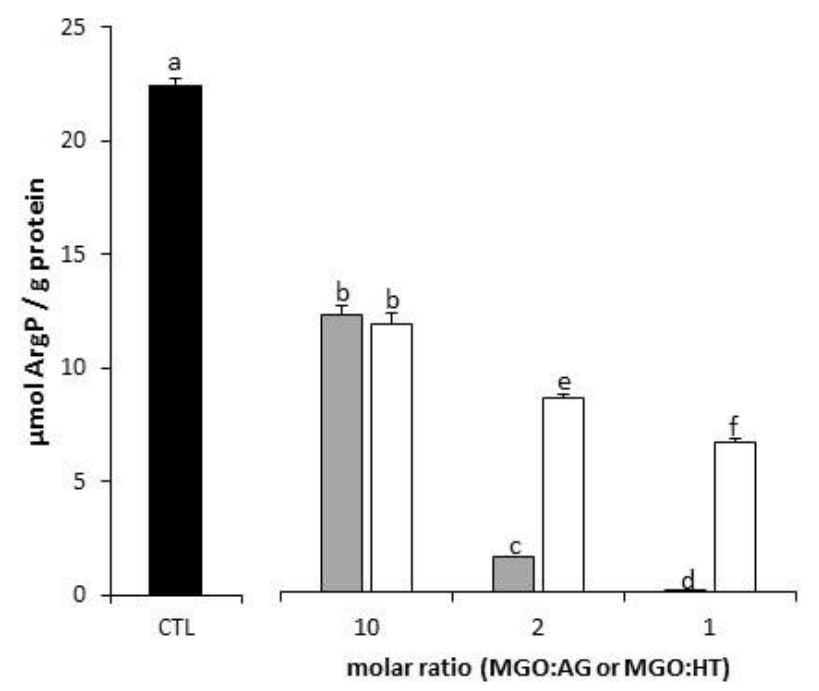

